\title{
ANALYSIS OF PRESSURE VARIATION OF FLUID IN AN INFINITE ACTING RESERVOIR
}

\author{
I. D. Erhunmwun ${ }^{1,}$, and J. A. Akpobi ${ }^{2}$ \\ 1,2 Department of Production Engineering, University of Benin, Benin City, Edo State, Nigeria \\ E-mail addresses:1 iredia.erhunmwun@uniben.edu, 2john.akpobi@uniben.edu
}

\begin{abstract}
In this work, we investigate the well pressure distribution in a boundless reservoir. This research work seeks to know the pressure variation in a reservoir whose pressure disturbance caused by the withdrawal of fluid from the wellbore, is yet to be felt at the external boundaries of the reservoir. The diffusivity equation was used in the analysis. The work covers the transient state where the reservoir is acting as if it was infinite in size. The finite element technique, using Lagrange quadratic shape elements was employed to carry out the analysis over the cross-section of the reservoir. The analysis was done with the assumption that before the well begins production, there was uniform distribution of pressure all through the reservoir. The accuracy of the result was validated by comparing with the results published by Chatas and Lee. The comparison shows a strong positive correlation between the two methods. Also, the results published by Chatas and Lee stated only the pressure at the wellbore at a particular time but this work predicts the pressure variation in the entire reservoir from the wellbore to the infinite sized external boundary at the same time.
\end{abstract}

Keywords: Infinite acting reservoir, permeability, porosity, dimensionless variables, diffusivity equation, and CrankNicholson scheme.

$\begin{array}{ll}\text { Nomenclature } \\ B & \text { Formation volume factor, RB/STB } \\ c & \text { Compressibility, psia }{ }^{-1} \\ h & \text { Thickness, } \mathrm{ft} \\ K & \text { Stiffness matrix } \\ k & \text { Permeability, md } \\ M & \text { Mass matrix } \\ n & \text { Number of elements } \\ P & \text { Pressure, psi } \\ P_{D} & \text { Dimensionless pressure } \\ P_{D} & \text { Dimensionless pressure rate } \\ P_{i} & \text { Initial reservoir pressure, psi } \\ Q & \text { Terminal flow rate } \\ q & \text { Volumetric flow rate, STB/D } \\ r & \text { Radius, ft } \\ r_{D} & \text { Dimensionless radius }\end{array}$

\section{INTRODUCTION}

There are basically three types of flow regimes that exist in describing the flow behaviour of fluids and pressure distribution as a function of time in reservoirs. These flow regimes are: steady-state flow, pseudo-steady-state flow, and unsteady-state flow [1].

The unsteady-state flow frequently called transient flow is a fluid flow condition that occurs when the rate of

$\begin{array}{ll}r_{e} & \text { External radius, } \mathrm{ft} \\ r_{e D} & \text { Dimensionless external radius } \\ r_{w} & \text { Wellbore radius, } \mathrm{ft} \\ s & \text { Time step, hr } \\ t & \text { Time, hr } \\ t_{D} & \text { Dimensionless time } \\ w & \text { Weight function } \\ \forall & \text { For all } \\ \mathrm{Greek} \text { letters } \\ \Delta t & \text { Time increment, hr } \\ \alpha & \text { Family of approximation } \\ \phi & \text { Porosity, fraction } \\ \mu & \text { Viscosity, cp } \\ \pi & \text { Pi } \\ \psi & \text { Interpolation function } \\ \infty & \text { Infinite }\end{array}$

change of pressure with respect to time at any position in the reservoir is neither zero nor constant. This definition suggests that the pressure derivative with respect to time is essentially a function of both position and time. In the unsteady-state flow cases, it is assumed that a well is located in a very large reservoir and producing at a constant flow rate. This rate creates a pressure disturbance in the reservoir that travels throughout this 
infinite-size reservoir. During this transient flow period, reservoir boundaries have no effect on the pressure behaviour and this is often very short in duration.

The constant terminal rate is one of the solutions of the radial diffusivity equation for a reservoir acting as if it was infinite in size and where necessary, they are applied in majority of well test analysis techniques. This, in itself, presents problems because for any second-order differential equation there is an infinite number of a possible solution, dependent on the choice of initial and boundary conditions [2].

Transient pressure response for a well producing from a finite reservoir of circular, square, and rectangular drainage shapes has been studied by [3], [4], [5], [6], [7], [8], [9] and [10]among others. Van Everdingen and Hurst presented the solution to diffusivity equation in eq. 8 in the form of infinite series of exponential terms and Bessel functions. The authors evaluated this series for several values of $r_{e D}$ over a wide range of values for $t_{D}$. [11] and [12]conveniently tabulated these solutions for the following two cases: Infinite-acting reservoir and Finite-radial reservoir.

Flows through porous media have been analysed in various ways through the years past. In well testing analysis, there are four solutions that are useful: the solution for a bounded circular reservoir: the solution for steady state case; the pseudo-steady state solution; and the solution that includes wellbore radius for a well in an infinite reservoir. This work addresses the case of a fluid in in an infinite sized reservoir.

\section{THEORY}

The law of conservation of mass, Darcy's law and the equation of state has been combined to obtain the following partial differential equation:

$$
\frac{\partial^{2} P}{\partial r^{2}}+\frac{1}{r} \frac{\partial P}{\partial r}=\frac{\emptyset \mu c}{0.000264 k} \frac{\partial P}{\partial t}
$$

with the assumptions that compressibility, $c$ is small and independent of pressure, P; permeability, $\mathrm{k}$, is constant and isotropic; viscosity, $\mu$, is independent of pressure; porosity, $\phi$, is constant; and that certain terms in the basic differential equation (involving pressure gradients squared) are negligible.

In this work, the diffusivity equation was analysed for circular reservoirs, the case in which the well is located in the centre of a cylindrical reservoir with an infinite external boundary.

\section{GOVERNING EQUATION}

$$
\frac{\partial^{2} P}{\partial r^{2}}+\frac{1}{r} \frac{\partial P}{\partial r}=\frac{\emptyset \mu c}{0.000264 k} \frac{\partial P}{\partial t}
$$

Initial and boundary conditions:

$$
\text { i. } P=P_{i} \text { at } \mathrm{t}=0 \forall r
$$

$$
\begin{aligned}
& \text { ii. }\left(r \frac{\partial P}{\partial r}\right)_{r_{w}}=\frac{q^{B} \mu}{2 \pi k h} \text { for }>.0 \\
& \text { iii. }\left(r \frac{\partial P}{\partial r}\right)_{r_{w}}=0 \forall t
\end{aligned}
$$

\section{DIMENSIONLESS VARIABLES}

The above equations incorporate physical parameters such as permeability, and it would be futile to solve this problem for a particular combination of values for these parameters. Dimensionless variables are designed to eliminate the physical parameters that affect quantitatively, but not qualitatively, the reservoir response. The above equations are in Darcy units, and the dimensionless terms will render the system of units employed irrelevant. For this line source model, 3 dimensionless variables are required. In US Oilfield units, distance, time and pressure are replaced as follows:

Dimensionless time:

$$
T_{D}=\frac{0.0002637 k t}{\emptyset \mu c r_{w}^{2}}
$$

Dimensionless distance:

$$
r_{D}=\frac{r}{r_{w}}
$$

Dimensionless pressure:

$$
P_{D}=\frac{k h}{141.2_{q} B_{\mu}}\left(P_{1}-P\right)
$$

By defining dimensionless pressure and dimensionless time in this way, it is possible to create an analytical model of the well and reservoir, or theoretical 'typecurve', that provides a 'global' description of the pressure response that is independent of the flow rate or of the actual values of the well and reservoir parameters.

Eq.1 can be transformed by substituting the following dimensionless variables in Eqns. 5-7 into eq. 1 and this becomes:

$$
\frac{\partial^{2} P_{D}}{\partial r_{D}{ }^{2}}+\frac{1}{r_{D}} \frac{\partial P_{D}}{\partial r_{D}}=\frac{\partial P_{D}}{\partial t_{D}}
$$

and the boundary and initial conditions become:

1. Dimensionless initial condition (uniform pressures in the reservoir):

$$
P_{D}\left(r_{D}, t_{D}=0\right)
$$

2. Dimensionless inner boundary condition (constant rate at the well):

$$
\frac{\partial P_{D}}{\partial r_{D}}\left(1, t_{D}\right)=-1
$$

3. Dimensionless Outer Boundary Conditions (Infinite acting reservoirs):

$$
P_{D}=\left(r_{D} \rightarrow \infty, t_{D}\right)=0
$$

\section{ASSUMPTIONS}

The assumptions used in proposing a solution to the diffusivity equation are as follows:

- The well is producing at constant flow rate.

- The reservoir is at uniform pressure, $P_{i}$ when production begins.

Vol. 36, No. 1 January 2017 
- $\quad$ The well, with a wellbore radius of $r_{W}$ is centred in a cylindrical reservoir of radius $r_{e D}$.

- No flow across the outer boundary, i.e., at r.

- The diffusivity equation was analysed for boundless circular reservoirs.

\section{FINITE ELEMENT FORMULATION}

a. Weak Formulation

In the development of the weak form, we assumed a quadratic element mesh and placed it over the domain and applied the following steps:

From eq. 8, we have:

$$
\frac{\partial P_{D}}{\partial t_{D}}+\frac{1}{r_{D}} \frac{\partial}{\partial r_{D}}\left(r \frac{\partial P_{D}}{\partial r_{D}}\right)=0
$$

Multiply eq. 12 by the weight $w$ function and integrate the final equation over the domain.

$$
\int_{v} w\left[\frac{\partial P_{D}}{\partial t_{D}}+\frac{1}{r_{D}} \frac{\partial}{\partial r_{D}}\left(r_{D} \frac{\partial P_{D}}{\partial r_{D}}\right)\right] d v=0
$$

Eq. 13 becomes,

$$
\int_{0}^{1} \int_{0}^{2 \pi r} \int_{r_{D A}}^{D B} w\left[\frac{\partial P_{D}}{\partial t_{D}}+\frac{1}{r_{D}} \frac{\partial}{\partial r_{D}}\left(r_{D} \frac{\partial P_{D}}{\partial r_{D}}\right)\right] r_{D} d r_{D} d \theta d z=0
$$

Integrating eq. 14 with respect to $z$, then $\theta$, over the limits, we have:

$$
\int_{r_{D A}}^{r_{D B}} w\left[\frac{\partial P_{D}}{\partial t_{D}}+\frac{1}{r_{D}} \frac{\partial}{\partial r_{D}}\left(r_{D} \frac{\partial P_{D}}{\partial r_{D}}\right)\right] r_{D} d r_{D}=0
$$

Eq. 15 can be exploded into:

$$
\int_{r_{D A}}^{r_{D B}} w \frac{\partial P_{D}}{\partial t_{D}} r_{D} d r_{D}-\int_{r_{D A}}^{r_{D B}} w \frac{\partial}{\partial r_{D}}\left(r_{D} \frac{\partial P_{D}}{\partial r_{D}}\right) d r_{D}=0
$$

Integrating eq. 16 by part, we have:

$$
\begin{gathered}
\int_{r_{D A}}^{r_{D B}} r_{D} \frac{\partial w}{\partial r_{D}} \frac{\partial P_{D}}{\partial r_{D}} d r_{D}-w\left[r_{D} \frac{\partial P_{D}}{\partial r_{D}}\right]_{r_{D A}}^{r_{D B}}+\int_{r_{D A}}^{r_{D B}} r_{D} w \frac{\partial P_{D}}{\partial t_{D}} d r_{D} \\
=0
\end{gathered}
$$

Grouping eq. 17 into linear and bilinear components, we have:

$$
\begin{gathered}
\int_{r_{D A}}^{r_{D B}} r_{D} \frac{\partial w}{\partial r_{D}} \frac{\partial P_{D}}{\partial r_{D}} d r_{D}+\int_{r_{D A}}^{r_{D B}} r_{D} w \frac{\partial P_{D}}{\partial t_{D}} d r_{D}-w\left[r_{D} \frac{\partial P_{D}}{\partial r_{D}}\right]_{r_{D A}}^{r_{D B}} \\
=0 \\
\int_{r_{D A}}^{r_{D B}} r_{D} \frac{\partial w}{\partial r_{D}} \frac{\partial P_{D}}{\partial r_{D}} d r_{D}+\int_{r_{D A}}^{r_{D B}} r_{D} w \frac{\partial P_{D}}{\partial t_{D}} d r_{D}-w Q_{A}-w Q_{B} \\
\text { Where } Q=0
\end{gathered}
$$

b. Interpolation Function

The weak form in eq. 19 requires that the approximation chosen for $P_{D}$ should be at least quadratic in $r_{D}$ so that there are no terms in eq. 19 that are identically zero.
Since the primary variable is simply the function itself, the Lagrange family of interpolation functions is admissible. We proposed that $P_{D}$ is the approximation over a typical finite element domain by the expression:

$$
P_{D}\left(r_{D}, t_{D}\right)=\sum_{j-1}^{n} P_{D j}\left(t_{D}\right) \psi_{j}^{e}\left(r_{D}\right) \text { and } w=\psi_{j}^{e}\left(r_{D}\right)
$$

Substituting eq. 20 into eq. 19, we have:

$$
\begin{gathered}
\int_{r_{D A}}^{r_{D B}} r_{D} \frac{d \psi_{j}^{e}}{d r_{D}} \frac{\partial}{d r_{D}} \sum_{j-1}^{n} P_{D j}\left(t_{D}\right) \psi_{j}^{e}\left(r_{D}\right) d r_{D} \\
+\int_{r_{D A}}^{r_{D B}} r_{D} \psi_{j}^{e} \frac{d}{d t_{D}} \sum_{j-1}^{n} P_{D j}\left(t_{D}\right) \psi_{j}^{e}\left(r_{D}\right) d r_{D} \\
-Q_{i}^{e}=0
\end{gathered}
$$

Factor out $\sum_{j-1}^{n} P_{D j}$

$$
\begin{gathered}
\sum_{j-1}^{n} P_{D j}+\int_{r_{D A}}^{r_{D B}} r_{D} \frac{d \psi_{i}^{e}}{d r_{D}} \frac{d \psi_{j}^{e}}{d r_{D}} d r_{D}+\sum_{j-1}^{n} \dot{P}_{D j} \int_{r_{D A}}^{r_{D B}} r_{D} \psi_{i}^{e} \psi_{j}^{e} d r_{D} \\
-Q_{i}^{e}=0 \\
\text { where } \dot{P}_{D j}=\frac{d P_{D}}{d t_{D}}
\end{gathered}
$$

In matrix form we can represent the semi-discrete finite element model thus,

$$
\left|K_{i j}^{e}\right|\left\{P_{D}\right\}+\left|M_{i j}^{e}\right|\left\{\dot{P}_{D j}\right\}=\left\{Q_{i}^{e}\right\}
$$

Eq. (23) is known as the finite element model.

Where

$$
\begin{gathered}
K_{i j}^{e}=\int_{r_{D A}}^{r_{D B}} r_{D} \frac{d \psi_{i}^{e}}{d r_{D}} \frac{d \psi_{j}^{e}}{d r_{D}} d r_{D} \\
M_{i j}^{e}=\int_{r_{D A}}^{r_{D B}} r_{D} \psi_{i}^{e} \psi_{j}^{e} d r_{D}
\end{gathered}
$$

Using Quadratic Lagrange Interpolation functions for a quadratic element:

$$
\begin{gathered}
\psi_{1}(r)=\frac{1}{h^{2}}\left(h+r_{A}-r\right)\left(h-2 r+2 r_{A}\right) \\
\psi_{2}(r)=\frac{4}{h^{2}}\left(r-r_{A}\right)\left(h+r_{A}-r\right) \\
\psi_{3}(r)=\frac{-1}{h^{2}}\left(r-r_{A}\right)\left(h-2 r+2 r_{A}\right)
\end{gathered}
$$

The coefficient matrix can be easily derived by substituting the Lagrange interpolation functions into eq. 24 respectively. The matrices are shown below: $\left[K^{e}\right]$

$$
=\frac{1}{6 h}\left[\begin{array}{ccc}
3 h+14 r_{A} & -\left(4 h+16 r_{A}\right) & h+2 r_{A} \\
-\left(4 h+16 r_{A}\right) & 16 h+32 r_{A} & -\left(12 h+16 r_{A}\right) \\
h+2 r_{A} & -\left(12 h+16 r_{A}\right) & 11 h+14 r_{A}
\end{array}\right]
$$

Also, the mass matrices can be easily derived by substituting the Lagrange interpolation functions into eq. 25 respectively. The matrices are shown below:

$\left[M^{e}\right]=\frac{1}{60}\left[\begin{array}{ccc}h+8 r_{A} & 4 r_{A} & -h-2 r_{A} \\ 4 r_{A} & 16 h+32 r_{A} & 4 h+4 r_{A} \\ -h-2 r_{A} & 4 h+4 r_{A} & 7 h+8 r_{A}\end{array}\right]$ 
Using four quadratic elements,

$$
r_{A}=r_{w}+(n-1) h
$$

In this analysis, we have withheld the computational details of the shape assembly of the finite element analysis (FEA) used. However, the authors would be glad to interact with researchers who may want to refer to the computational mathematics involved.

\section{TIME APPROXIMATION}

Recalling eq. 23: $\left|K_{i j}^{e}\right|\left\{P_{D}\right\}+\left|M_{i j}^{e}\right|\left\{\dot{P}_{D j}\right\}=\left\{Q_{i}^{e}\right\}$

For a given time step s, eq. 23 becomes

$$
\left|K_{i j}^{e}\right|\left\{P_{D}\right\}_{s}+\left|M_{i j}^{e}\right|\left\{\dot{P}_{D j}\right\}_{s}=\left\{Q_{i}^{e}\right\}_{s}
$$

For the next time step $s+1$, eq. 23 becomes

$$
\left|K_{i j}^{e}\right|\left\{P_{D}\right\}_{s+1}+\left|M_{i j}^{e}\right|\left\{\dot{P}_{D j}\right\}_{s+1}=\left\{Q_{i}^{e}\right\}_{s+1}
$$

Multiply eq. 32 by $(1-\alpha)$ and eq. 33 by $\alpha$, then we add the two resulting equations,

$$
\begin{aligned}
{\left[M_{i j}^{e}\right]\left[(1-a)\left\{\dot{P}_{D j}\right\}_{s}+a\left\{\dot{P}_{D j}\right\}_{s+1}\right] } \\
+\left[K_{i j}^{e}\right]\left[(1-a)\left\{P_{D j}\right\}_{s+1}+a\left\{P_{D j}\right\}_{s+1}\right] \\
=(1-a)\left\{Q_{i}^{e}\right\}_{s+1}
\end{aligned}
$$

The $\alpha$ family of interpolation for time consideration is given as:

$$
(1-a)\left\{\dot{P}_{D j}\right\}_{s}+a\left\{\dot{P}_{D j}\right\}_{s+1}=\frac{\left\{P_{D j}\right\}_{s+1}-\left\{P_{D j}\right\}_{s}}{\Delta t_{s+1}}
$$

Substitute eq. 35 into eq. 34 and using the CrankNicholson Scheme where, $\alpha=1 / 2$

$$
\begin{aligned}
{\left[\left[M_{i j}^{e}\right]+\frac{\Delta t_{s+1}}{2}\left[K_{i j}^{e}\right]\right]\left\{P_{D j}\right\}_{s+1} } & \\
= & {\left[\left[M_{i j}^{e}\right]-\frac{\Delta t_{s+1}}{2}\left[K_{i j}^{e}\right]\right]\left\{P_{D j}\right\}_{s} } \\
& +\frac{\Delta t_{s+1}}{2}\left[\left\{Q_{i}^{e}\right\}_{s}+\left\{Q_{i}^{e}\right\}_{s+1}\right]
\end{aligned}
$$

From the initial condition given in eq. 9 for a constant terminal rate case, it implies that when $s=0$, i.e., initial time, all dimensionless pressure in the reservoir will be zero. Also, the flow rate was constant all through operation. This means that $\left\{Q_{i}^{e}\right\}_{s}+\left\{Q_{i}^{e}\right\}_{s+1}$. Hence, eq. 36 becomes:

$$
\begin{aligned}
{\left[\left[M_{i j}^{e}\right]+\frac{\Delta t_{s+1}}{2}\left[K_{i j}^{e}\right]\right]\left\{P_{D j}\right\}_{1} } & \\
= & {\left[\left[M_{i j}^{e}\right]-\frac{\Delta t_{1}}{2}\left[K_{i j}^{e}\right]\left\{P_{D j}\right\}_{0}\right.} \\
& \left.+\Delta t_{1}\left\{\bar{Q}_{i}^{e}\right\}\right]
\end{aligned}
$$

Where $\bar{Q}_{i}^{e}=\frac{1}{2}\left(Q_{i}^{1}\right)_{s+1}+\frac{1}{2}\left(Q_{i}^{1}\right)_{s}$

$$
\begin{aligned}
\left\{P_{D j}\right\}_{1}=\left[\left[M_{i j}^{e}\right]+\right. & \left.\frac{\Delta t_{1}}{2}\left[K_{i j}^{e}\right]\right]^{-1}\left[\left[M_{i j}^{e}\right]-\frac{\Delta t_{1}}{2}\left[K_{i j}^{e}\right]\right]\left\{P_{D j}\right\}_{0} \\
& +\Delta t_{1}\left\{\bar{Q}_{i}^{e}\right\}
\end{aligned}
$$

\section{RESULTS AND DISCUSSION}

When a well is put on production with the assumption that the well is producing at a constant flow rate after a shut-in period, the pressure in the wellbore begins to drop due to the withdrawal of the fluid from the reservoir through the well bore. This drop in pressure at the well bore causes a pressure disturbance that spreads through the entire reservoir. The influence of the reservoir boundaries or the shape of the drainage area at this point does not have any effect on the rate at which the pressure disturbance spreads in the formation. That is why the transient state flow is also called the infinite acting state. During this period, the external boundary of the reservoir is assumed mathematically to be at infinity i.e., $r_{e D} \rightarrow \infty$. This means that the value of the dimensionless pressure $P_{D}$ is not affected by the external reservoir boundary but only a function of the dimensionless time $t_{D}$. At this point, it is important to note that in actual sense, the external boundary of the reservoir is not at infinity. During the infinite acting period, the declining rate of wellbore pressure and the manner by which the pressure disturbance spreads through the reservoir are determined by reservoir and fluid characteristics. These characteristics include porosity, $\phi$ permeability, $\mathrm{k}$, total compressibility, $c_{t}$ and the fluid viscosity, $\mu$.

Graphs of dimensionless pressure against dimensionless time from results obtained from the FEM analysis in the infinite acting reservoirs are shown in Figs. 1 and 2. These were shown for different radial values of dimensionless external radius of 1.5 and 4 and dimensionless times step of 0.0005 and 0.01 respectively. Presented in Fig 1 is the dimensionless pressure between dimensionless time of zero and 0.1 and at time step of 0.0005 and Fig 2 presents the dimensionless pressure between dimensionless time of zero and 3. From Figs. 1 and 2, it was observed that before the reservoir starts production, i.e., $t_{D}=0$, the dimensionless pressure was zero in the entire reservoir. This means that before production, the initial pressure in the entire reservoir was constant. As production begins, the dimensionless pressure increases drastically at the early stage. This sudden increase in the dimensionless pressure was as a result of the withdrawal of fluid from the reservoir. Later on, the dimensionless pressure increases almost uniformly with a small slope. This is due to the fact that the fluid which has been withdrawn from the reservoir has been recharged mainly because of the expansion of the compressed fluid within the reservoir. 


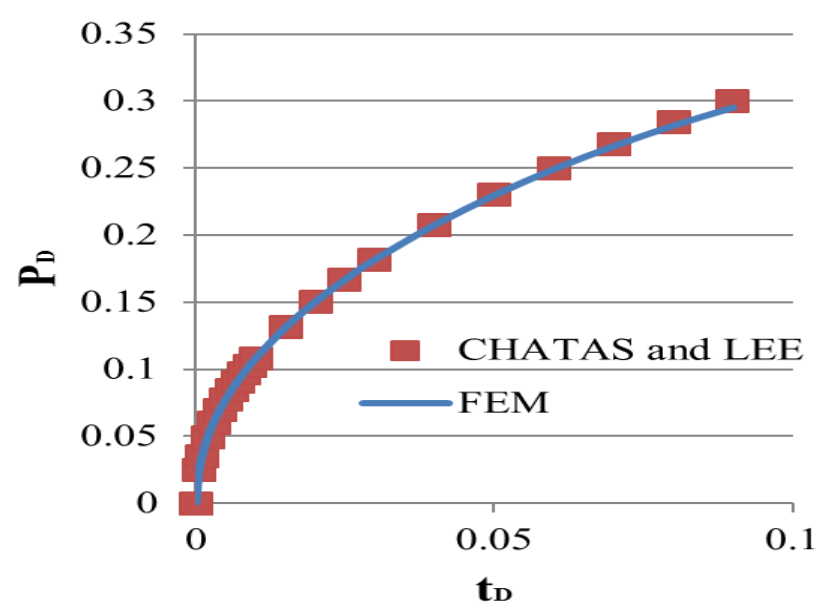

Fig. 1: A graph of PD against tD for FEM and Chatas and Lee results in the infinite reservoirs for $r_{e D}=1.5$ and $\Delta t=$ 0.0005

Table 2: Comparison between FEM and Chatas and Lee results in infinite acting reservoirs for $r_{e D}=4$ and $\Delta t=$ 0.01

\begin{tabular}{cccc}
\hline$t D$ & \% error & tD & \% error \\
\hline 0.15 & 0.1333 & 0.8 & 0.0677 \\
0.20 & 0.0707 & 0.9 & 0.0389 \\
0.30 & 0.0398 & 1.0 & 0.0249 \\
0.40 & 0.0354 & 1.2 & 1.2685 \\
0.50 & 0.0324 & 1.4 & 1.2991 \\
0.60 & 0.0604 & 2.0 & 0.0196 \\
0.70 & 0.0569 & 3.0 & 1.2602 \\
\hline
\end{tabular}

Table 1: Comparison between FEM and Chatas and Lee results in infinite acting reservoirs for $r_{e D}=1.5$ and $\Delta t=$ 0.0005

\begin{tabular}{rrrrrr}
\hline Td & \% error & tD & \% error & tD & \% error \\
\hline 0.0000 & 0.0000 & 0.007 & 0.1098 & 0.04 & 0.0481 \\
0.0005 & 6.0000 & 0.008 & 0.1030 & 0.05 & 0.1304 \\
0.0010 & 0.8523 & 0.009 & 0.0973 & 0.06 & 0.2800 \\
0.0020 & 0.6061 & 0.010 & 0.0925 & 0.07 & 0.5970 \\
0.0030 & 0.6633 & 0.015 & 0.0000 & 0.08 & 0.9842 \\
0.0040 & 0.5764 & 0.020 & 0.0000 & 0.09 & 1.5672 \\
0.0050 & 0.2584 & 0.025 & 0.0000 & 0.10 & 2.2583 \\
0.006 & 0.2367 & 0.03 & 0.0000 & & \\
\hline
\end{tabular}

The results presented in Figs 1 and 2 are the dimensionless pressure at the well bore of the reservoir. The result from the FEM analysis was seen to agree with results publish by Chatas and Lee. To test for the level of convergence of the results, a table of percentage error between the FEM solution and the results published by Chatas and Lee was generated. This was shown in Table 1 with dimensionless external boundary radius of 1.5 and time step of 0.0005 and Table 2 with dimensionless external boundary radius of 4 and time step of 0.01 .

Dimensionless Pressure against Dimensionless Radial Displacement

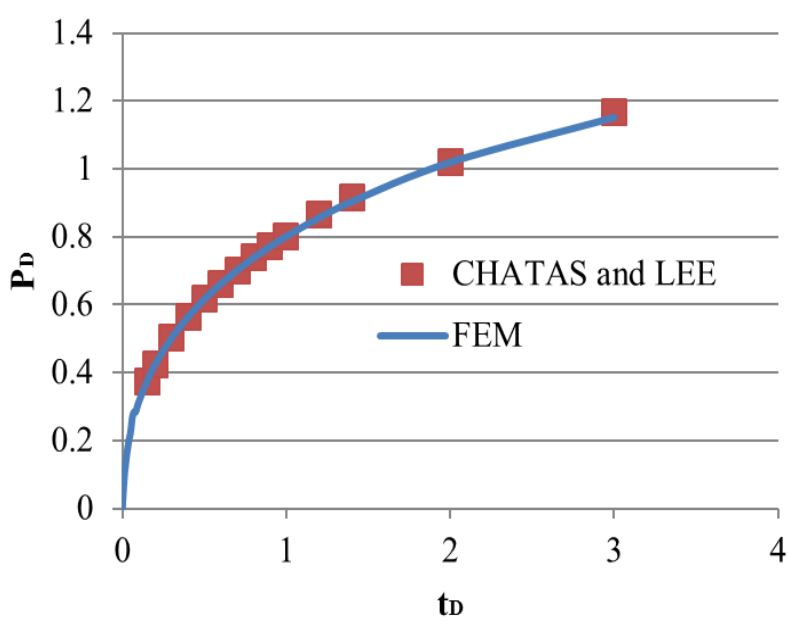

Fig. 2: A graph of PD against tD for FEM and Chatas and Lee results in the infinite reservoirs for $r_{e D}=4$ and $\Delta t=0.01$

At this point, it is pertinent to note that irrespective of the fact that there is a change in dimensionless pressure with dimensionless time at the well bore which has been the main course of many researchers. It will be important to note also that for a particular dimensionless time, there is a corresponding change in the dimensionless pressure with the dimensionless radial displacement. So, at the transient period where the reservoir is acting as if it is infinite in size, there is pressure variation within the reservoir.

Table 3 shows the dimensionless pressure $\left(P_{D}\right)$ variation throughout the reservoir. It was observed that $P_{D}$ values decreases from the well bore out into the reservoir until the infinite sized reservoir boundary where $P_{D}=0$. It was also observed that there were times within the reservoir where the pressure at some points within the reservoir was greater than the initial pressure of the reservoir. This is shown by the negative $P_{D}$ values in Tables 3 and 4 . These negative $P_{D}$ values where seen to occur at the early stage of the transient period in the infinite acting reservoir. Thereafter, $P_{D}$ values become positive all through the infinite sized reservoir. From this analysis, Table 3 shows the results obtained for a well whose dimensionless external boundary radius is 1.5 but acting as if it was acting infinite in size and the time step is 0.0005 .

Also, Table 4 also shows the results obtained from the FEM analysis but at this point, the dimensionless external boundary radius was 4 and the time step was 0.01. At this point, the reservoir extent is yet to be felt. What this means is that the pressure disturbance is yet to be felt at the external boundary radius of the reservoir which in this case is 4 . This is the reason the $P_{D}$ values at the dimensionless external boundary radius of 1.5 and 4 were respectively zero. 
Table 3: Pressure Distribution in an infinite acting reservoir with $r e D=1.5$

\begin{tabular}{|c|c|c|c|c|c|c|c|c|c|}
\hline $\mathrm{rD}$ & 1.0000 & 1.0625 & 1.1250 & 1.1875 & 1.2500 & 1.3125 & 1.3750 & 1.4375 & 1.5000 \\
\hline $\mathrm{tD}$ & \multicolumn{9}{|c|}{ Dimensionless Pressure } \\
\hline 0.0005 & 0.0235 & -0.0009 & 0.0019 & $-7.30 \mathrm{E}-05$ & 0.0002 & $-6.00 \mathrm{E}-06$ & $1.26 \mathrm{E}-05$ & $-4.50 \mathrm{E}-07$ & 0.0000 \\
\hline 0.001 & 0.0349 & 0.0024 & 0.0007 & 0.0003 & -0.0001 & $2.92 \mathrm{E}-05$ & $-2.30 \mathrm{E}-05$ & $2.64 \mathrm{E}-06$ & 0.0000 \\
\hline 0.0015 & 0.0432 & 0.0063 & 0.0004 & 0.0003 & $-3.10 \mathrm{E}-05$ & $-1.10 \mathrm{E}-06$ & $7.18 \mathrm{E}-06$ & $-2.20 \mathrm{E}-06$ & 0.0000 \\
\hline 0.002 & 0.0498 & 0.0101 & 0.0008 & 0.0002 & $7.63 \mathrm{E}-05$ & $-8.00 \mathrm{E}-06$ & $1.29 \mathrm{E}-05$ & $-8.50 \mathrm{E}-07$ & 0.0000 \\
\hline 0.003 & 0.0607 & 0.0175 & 0.0029 & 0.0004 & 0.0002 & $2.21 \mathrm{E}-05$ & $2.38 \mathrm{E}-06$ & $2.06 \mathrm{E}-06$ & 0.0000 \\
\hline 0.004 & 0.0698 & 0.0243 & 0.0058 & 0.001 & 0.0002 & $5.44 \mathrm{E}-05$ & $7.82 \mathrm{E}-06$ & $1.53 \mathrm{E}-06$ & 0.0000 \\
\hline 0.005 & 0.0776 & 0.0307 & 0.0091 & 0.0021 & 0.0004 & $9.20 \mathrm{E}-05$ & $2.37 \mathrm{E}-05$ & $3.11 \mathrm{E}-06$ & 0.0000 \\
\hline 0.006 & 0.0847 & 0.0366 & 0.0126 & 0.0034 & 0.0008 & 0.0002 & $4.47 \mathrm{E}-05$ & $7.81 \mathrm{E}-06$ & 0.0000 \\
\hline 0.007 & 0.0912 & 0.0422 & 0.0161 & 0.005 & 0.0013 & 0.0003 & 7.57E-05 & $1.54 \mathrm{E}-05$ & 0.0000 \\
\hline 0.008 & 0.0972 & 0.0475 & 0.0197 & 0.0069 & 0.002 & 0.0005 & 0.0001 & $2.71 \mathrm{E}-05$ & 0.0000 \\
\hline 0.009 & 0.1029 & 0.0525 & 0.0232 & 0.0088 & 0.0028 & 0.0008 & 0.0002 & $4.58 \mathrm{E}-05$ & 0.0000 \\
\hline 0.01 & 0.1082 & 0.0573 & 0.0267 & 0.0108 & 0.0038 & 0.0012 & 0.0003 & $7.51 \mathrm{E}-05$ & 0.0000 \\
\hline 0.015 & 0.1312 & 0.0784 & 0.0432 & 0.0218 & 0.001 & 0.0041 & 0.0015 & 0.0005 & 0.0000 \\
\hline 0.02 & 0.1503 & 0.0964 & 0.0582 & 0.0329 & 0.0174 & 0.0085 & 0.0038 & 0.0014 & 0.0000 \\
\hline 0.025 & 0.1669 & 0.1123 & 0.072 & 0.0438 & 0.0253 & 0.0137 & 0.0068 & 0.0027 & 0.0000 \\
\hline 0.03 & 0.1818 & 0.1265 & 0.0846 & 0.0543 & 0.0332 & 0.0192 & 0.0102 & 0.0043 & 0.0000 \\
\hline 0.035 & 0.1952 & 0.1395 & 0.0964 & 0.0642 & 0.0411 & 0.0249 & 0.0139 & 0.0061 & 0.0000 \\
\hline 0.04 & 0.2076 & 0.1515 & 0.1074 & 0.0737 & 0.0487 & 0.0306 & 0.0175 & 0.0079 & 0.0000 \\
\hline 0.045 & 0.2191 & 0.1627 & 0.1177 & 0.0826 & 0.056 & 0.036 & 0.0212 & 0.0097 & 0.0000 \\
\hline 0.05 & 0.2298 & 0.1731 & 0.1273 & 0.0911 & 0.0629 & 0.0413 & 0.0247 & 0.0114 & 0.0000 \\
\hline 0.055 & 0.2398 & 0.1829 & 0.1364 & 0.0991 & 0.0696 & 0.0464 & 0.0281 & 0.0131 & 0.0000 \\
\hline 0.06 & 0.2493 & 0.1921 & 0.145 & 0.1066 & 0.0759 & 0.0512 & 0.0313 & 0.0147 & 0.0000 \\
\hline 0.065 & 0.2581 & 0.2007 & 0.153 & 0.1138 & 0.0818 & 0.0558 & 0.0344 & 0.0162 & 0.0000 \\
\hline 0.07 & 0.2664 & 0.2089 & 0.1606 & 0.1205 & 0.0874 & 0.0602 & 0.0373 & 0.0177 & 0.0000 \\
\hline 0.075 & 0.2743 & 0.2166 & 0.1678 & 0.1269 & 0.0928 & 0.0643 & 0.0401 & 0.0191 & 0.0000 \\
\hline 0.08 & 0.2817 & 0.2238 & 0.1745 & 0.1329 & 0.0978 & 0.0681 & 0.0427 & 0.0204 & 0.0000 \\
\hline 0.085 & 0.2886 & 0.2306 & 0.1809 & 0.1385 & 0.1025 & 0.0718 & 0.0452 & 0.0217 & 0.0000 \\
\hline 0.09 & 0.2952 & 0.237 & 0.1869 & 0.1439 & 0.107 & 0.0753 & 0.0476 & 0.0228 & 0.0000 \\
\hline 0.095 & 0.3014 & 0.2431 & 0.1926 & 0.1489 & 0.1113 & 0.0786 & 0.0498 & 0.0239 & 0.0000 \\
\hline 0.1 & 0.3073 & 0.2488 & 0.1979 & 0.1537 & 0.1153 & 0.0816 & 0.0519 & 0.025 & 0.0000 \\
\hline
\end{tabular}

Table 4: Pressure Distribution in an infinite acting reservoir with reD $=4$

\begin{tabular}{|c|c|c|c|c|c|c|c|c|}
\hline $\mathrm{rD}$ & 1.0000 & 1.3750 & 1.7500 & 2.5000 & 2.8750 & 3.2500 & 3.6250 & 4.0000 \\
\hline tD & \multicolumn{8}{|c|}{ Dimensionless Pressure } \\
\hline 0.01 & 0.0813 & -0.0046 & 0.0071 & 0.0007 & $-4.80 \mathrm{E}-05$ & $6.66 \mathrm{E}-05$ & $-4.30 \mathrm{E}-06$ & 0.0000 \\
\hline 0.02 & 0.1336 & $5.96 \mathrm{E}-05$ & 0.0064 & 0.00011 & $6.18 \mathrm{E}-05$ & $-3.80 \mathrm{E}-05$ & $8.26 \mathrm{E}-06$ & 0.0000 \\
\hline 0.03 & 0.1715 & 0.0085 & 0.0043 & -0.0002 & 7.97E-05 & $-5.20 \mathrm{E}-05$ & $4.92 \mathrm{E}-06$ & 0.0000 \\
\hline 0.04 & 0.2013 & 0.0183 & 0.0027 & -0.0003 & $4.26 \mathrm{E}-05$ & $-1.80 \mathrm{E}-05$ & $-2.10 \mathrm{E}-06$ & 0.0000 \\
\hline 0.05 & 0.2262 & 0.0286 & 0.0021 & -0.0001 & $1.26 \mathrm{E}-06$ & $1.71 \mathrm{E}-05$ & $-5.30 \mathrm{E}-06$ & 0.0000 \\
\hline 0.06 & 0.2477 & 0.039 & 0.0024 & 8.64E-05 & $-2.10 \mathrm{E}-05$ & $3.48 \mathrm{E}-05$ & $-4.30 \mathrm{E}-06$ & 0.0000 \\
\hline 0.07 & 0.2667 & 0.0493 & 0.0035 & 0.0003 & $-2.00 \mathrm{E}-05$ & $3.64 \mathrm{E}-05$ & $-9.70 \mathrm{E}-07$ & 0.0000 \\
\hline 0.08 & 0.2839 & 0.0594 & 0.0053 & 0.0004 & $-8.60 \mathrm{E}-07$ & $2.90 \mathrm{E}-05$ & $2.48 \mathrm{E}-06$ & 0.0000 \\
\hline 0.09 & 0.2997 & 0.0692 & 0.0077 & 0.0005 & $2.87 \mathrm{E}-05$ & $1.93 \mathrm{E}-05$ & $4.94 \mathrm{E}-06$ & 0.0000 \\
\hline 0.10 & 0.3143 & 0.0788 & 0.0105 & 0.0006 & $6.28 \mathrm{E}-05$ & $1.18 \mathrm{E}-05$ & $6.11 \mathrm{E}-06$ & 0.0000 \\
\hline 0.11 & 0.328 & 0.0881 & 0.0136 & 0.0007 & $9.75 \mathrm{E}-05$ & 8.68E-06 & $6.23 \mathrm{E}-06$ & 0.0000 \\
\hline 0.12 & 0.3408 & 0.0971 & 0.0169 & 0.0008 & 0.0001 & $1.05 \mathrm{E}-05$ & 5.77E-06 & 0.0000 \\
\hline 0.13 & 0.3529 & 0.1059 & 0.0205 & 0.0008 & 0.0002 & $1.67 \mathrm{E}-05$ & $5.24 \mathrm{E}-06$ & 0.0000 \\
\hline 0.14 & 0.3645 & 0.1145 & 0.0243 & 0.0009 & 0.0002 & $2.67 \mathrm{E}-05$ & $5.04 \mathrm{E}-06$ & 0.0000 \\
\hline 0.15 & 0.3755 & 0.1228 & 0.0281 & 0.0011 & 0.0002 & 3.93E-05 & $5.47 \mathrm{E}-06$ & 0.0000 \\
\hline 0.20 & 0.4244 & 0.1615 & 0.0484 & 0.0024 & 0.0005 & 0.0001 & $1.97 \mathrm{E}-05$ & 0.0000 \\
\hline 0.30 & 0.5022 & 0.2272 & 0.0895 & 0.0088 & 0.0022 & 0.0005 & 0.0001 & 0.0000 \\
\hline 0.40 & 0.5643 & 0.2819 & 0.1284 & 0.0191 & 0.0062 & 0.0018 & 0.0004 & 0.0000 \\
\hline 0.50 & 0.6165 & 0.3291 & 0.1643 & 0.0319 & 0.0122 & 0.0042 & 0.0012 & 0.0000 \\
\hline 0.60 & 0.6618 & 0.3708 & 0.1976 & 0.046 & 0.0198 & 0.0077 & 0.0025 & 0.0000 \\
\hline 0.70 & 0.702 & 0.4083 & 0.2284 & 0.0609 & 0.0285 & 0.0122 & 0.0043 & 0.0000 \\
\hline
\end{tabular}




\begin{tabular}{ccccccccc}
\hline $\mathrm{rD}$ & 1.0000 & 1.3750 & 1.7500 & 2.5000 & 2.8750 & 3.2500 & 3.6250 & 4.0000 \\
\hline 0.80 & 0.7382 & 0.4423 & 0.2571 & 0.076 & 0.0379 & 0.0174 & 0.0065 & 0.0000 \\
0.90 & 0.7713 & 0.4736 & 0.2839 & 0.0912 & 0.0479 & 0.0231 & 0.0091 & 0.0000 \\
1.00 & 0.8017 & 0.5025 & 0.3091 & 0.1061 & 0.058 & 0.0292 & 0.0118 & 0.0000 \\
1.20 & 0.8562 & 0.5546 & 0.3552 & 0.1352 & 0.0784 & 0.0418 & 0.0177 & 0.0000 \\
1.40 & 0.9041 & 0.6006 & 0.3965 & 0.1625 & 0.0982 & 0.0544 & 0.0237 & 0.0000 \\
2.00 & 1.0197 & 0.7124 & 0.4985 & 0.2334 & 0.1509 & 0.0888 & 0.0404 & 0.0000 \\
3.00 & 1.1518 & 0.8406 & 0.6166 & 0.3182 & 0.215 & 0.1312 & 0.061 & 0.0000 \\
\hline
\end{tabular}

\section{CONCLUSION}

In this work, we have formulated the Finite Element Model and the model was used to analyse the diffusivity equation which shows the pressure distribution across a circular reservoir for the constant terminal rate case in which the reservoir is acting as if it was infinite in size. The results obtained at the well bore at different dimensionless radius and time steps were shown in Figs 1 and 2 and its accuracy was shown in Tables 1 and 2 . Also presented were Tables 3 and 4 that shows the results obtained for the pressure distribution throughout the infinite sized reservoirs. It was seen that the dimensionless pressure decreases from the well bore to the external boundary. The results obtained from this analysis showed that there was a strong correlation with the results published by Chatas and Lee. Also, this work analysed the pressure variation through various points within the reservoir formation.

\section{REFERENCES}

[1] Tarek A. Reservoir Engineering Handbook. Second edition. Gulf Professional Publishing, London. 2001.

[2] Dake, L. P. The Practice of Reservoir Engineering (Revised Edition). First edition, Elsevier Science. 2001.
[3] Van Everdingen, A. F. and Hurst, W. The Application of the Laplace Transformation to Flow Problems in Reservoir. Trans. AIME 186, 305-324. 1949.

[4] Miller, C. C., Dyes, A. B., and Hutchinson, C. A., Jr. The Estimation of Permeability and Reservoir Pressure from Bottom-Hole Pressure Build-Up Characteristics. Trans. AIME189, 91-104. 1950.

[5] Aziz, K. and Flock, D. L. Unsteady State Gas Flow - Use of Drawdown Data in the Reduction of Gas Well Behaviour. J. Can. Pet. Tech., 2 (l), 9-15. 1963.

[6] Earlougher, R. C., Jr., Ramey, H. J., Jr., Miller, F. G., and Mueller, T. D. Pressure Distributions in Rectangular Reservoirs. J. Pet. Tech., 199-208. 1968.

[7] Ramey, H. J., Jr. and Cobb, W. M. A General Pressure Build up Theory for a Well in a Closed Drainage Area. J. Pet. Tech., 1493 -1505. 1971.

[8] Kumar, A. and Ramey, H. J., Jr. Well-Test Analysis for a Well in a Constant-Pressure Square. Soc. Pet. Eng. J., 107116. 1974.

[9] Cobb, W. M. and Smith, J. T. An Investigation of Pressure Build up Tests in Bounded Reservoirs," J. Pet. Tech. 1975.

[10] Chen, H. K. and Brigham, W. E. Pressure Build up for a Well with Storage and Skin in a Closed Square. J. Pet. Tech., 141-146. 1978.

[11] Chatas, A. T. A Practical Treatment of Non-steady-state Flow Problems in Reservoir Systems. J. Pet. Eng., B-44-56. 1953.

[12] John L. Well Testing, Soc. Pet. Eng. of AIME, New York. 1982. 